\title{
Design and synthesis of superhydrophobic carbon nanofiber composite coatings for terahertz frequency shielding and attenuation
}

\author{
Arindam Das, ${ }_{3}^{1}$ Constantine M. Megaridis, ${ }^{1, a)}$ Lei Liu, ${ }^{2, b)}$ Tao Wang, ${ }^{3}$ and \\ Abhijit Biswas $^{3}$ \\ ${ }^{1}$ Department of Mechanical and Industrial Engineering, University of Illinois at Chicago, Chicago, \\ Illinois 60607, USA \\ ${ }^{2}$ Department of Electrical Engineering, Advanced Diagnostics and Therapeutics, University of Notre Dame, \\ Notre Dame, Indiana 46556, USA \\ ${ }^{3}$ Department of Electrical Engineering, Center for Nano Science and Technology, University of Notre \\ Dame, Notre Dame, Indiana 46556, USA
}

(Received 22 December 2010; accepted 4 April 2011; published online 25 April 2011)

\begin{abstract}
We report design and synthesis of polymer-based large-area superhydrophobic carbon nanofiber (CNF) composite coatings for tunable electromagnetic interference shielding and attenuation in the terahertz $(\mathrm{THz})$ frequency regime. Such coatings with different $\mathrm{CNF} /$ polymer weight ratios are characterized by a frequency domain $\mathrm{THz}$ spectroscopy system. A maximum $\mathrm{THz}$ shielding effectiveness of $\sim 32 \mathrm{~dB}$ was measured in the examined frequency range of 570-630 GHz. Coating attenuation level varied with CNF loading. Two-dimensional distributions of power attenuation at $600 \mathrm{GHz}$ showed good spatial uniformity. The present composite coatings, in addition to their self-cleaning property, have high potential for advanced technology high-frequency applications. (C) 2011 American Institute of Physics. [doi:10.1063/1.3583523]
\end{abstract}

Electromagnetic interference (EMI) shielding properties of pure materials or composites containing conductive fillers have been studied extensively in the megahertz range as well as in the lower frequencies of the gigahertz range. Filamentous carbon materials due to their chemical inertness, low production costs and the relatively low particle loadings required for sufficiently large shielding effectiveness (SE), offer an attractive choice for EMI shielding applications. For example, polymer composites containing vapor-grown carbon nanofibers (CNFs) have been studied at frequencies 15 $\mathrm{MHz}-75 \mathrm{GHz}$, with maximum SE within this frequency zone around $30-50 \mathrm{~dB}$ for $1-3 \mathrm{~mm}$ thick samples. ${ }^{1} \mathrm{CNF}-$ loaded polymer composite coatings about $100 \mu \mathrm{m}$-thick displayed $\mathrm{SE}$ of up to $25 \mathrm{~dB}$ in the X-band $(8.2$ to $12.4 \mathrm{GHz}){ }^{2}$

In recent years, there has been unprecedented growth in the development of terahertz (THz) devices, circuits and systems due to their promising applications in astronomy, chemical analysis, biological sensing, imaging and security screening. ${ }^{3-7}$ With the ever increasing speed of the electronic circuits and systems, EMI shielding in the $\mathrm{THz}$ region is becoming more important. ${ }^{8,9}$ In addition, effective $\mathrm{THz}$ attenuation devices are required in many quasi-optical systems (e.g., THz spectroscopy and imaging). Therefore, innovations in materials and processes for EMI shielding and attenuation of $\mathrm{THz}$ electronic devices are of immense interest for advanced technology applications.

In this letter, we present design and synthesis of largearea $\mathrm{CNF} /$ polytetrafluoroethylene (PTFE) polymer composite coatings as effective attenuators of $\mathrm{THz}$ radiation and report measurements on their EMI SE in the $570-630 \mathrm{GHz}$ frequency range. The coatings are fabricated by a simple method of spraying dispersions of vapor-grown CNFs and submicron PTFE particles in a polymer blend solution of

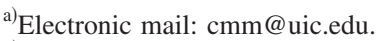

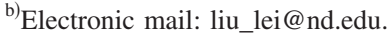

poly(vinylidene fluoride) (PVDF) and poly(methyl methacrylate) (PMMA) on cellulosic substrates. The procedure is inherently low-cost, and all ingredients are commercially available. The chemical inertness of the coatings along with their water repellency and self-cleaning ability prevent contamination and corrosion when exposed to outdoor conditions.

The low surface energy of the PVDF/PMMA blend used in the present coatings has a positive effect on the conductivity, and thus on the SE of these films. The presence of the PTFE particles in the coatings also contributes to conductivity through the volume exclusion effect. ${ }^{10}$

A solution blend of PVDF and PMMA forms the composite polymer matrix, which has a good degree of hydrophobicity and interfacial adhesion properties. Sub-micron PTFE particles are used as hydrophobic fillers to tune the coating microstructure and reduce surface energy, whereas the electrical conductivity is manipulated using heat-treated, vapor-grown CNFs, which have reduced amorphous carbon content and higher electrical conductivity compared to asgrown fibers. ${ }^{2}$ The amorphous chemical vapor deposition (CVD) carbon of as-grown fibers organizes in graphitized stacked cup and cone structures with heat treatment, in turn improving electrical conductivity. The CNFs used here were treated at $3000{ }^{\circ} \mathrm{C}$. High resolution transmission electron microscope (HRTEM) images of as-grown CNFs and their heat-treated derivatives are shown in Ref. 18.

The simultaneous presence of high conductivity and superhydrophobicity in these coatings bears promise for effective EMI shielding and other technologies requiring both extreme liquid repellency and high electrical conductivity.

To prepare the composite coatings, 60/40 wt \% solution blends of PVDF (530 kDa; Sigma-Aldrich, USA) and PMMA (996 kDa; Sigma-Aldrich, USA) were prepared by mixing $20 \mathrm{wt} \%$ solution of PVDF in dimethylformamide (DMF) with $10 \mathrm{wt} \%$ solution of PMMA in acetone. PTFE particles (average dia. $260 \pm 54.2 \mathrm{~nm}$; Sigma-Aldrich, USA) 
(a)

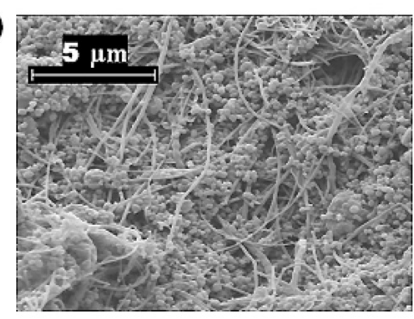

(b)

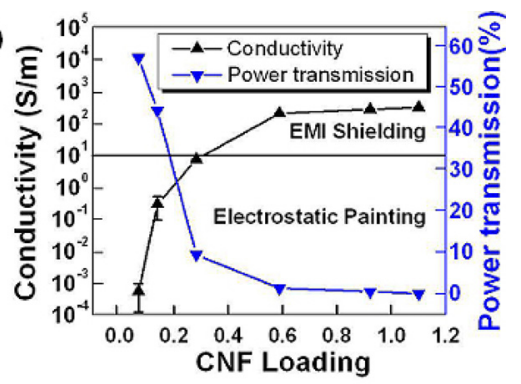

(c)

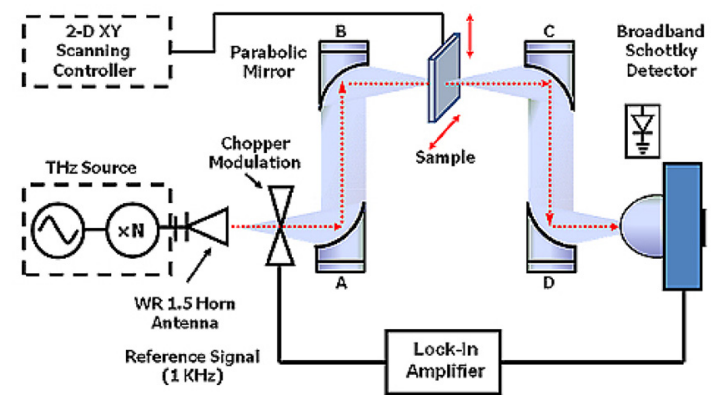

were dispersed by sonication in acetone at $0.2 \mathrm{wt} \%$, while CNFs (average fiber dia. $100 \mathrm{~nm}$, PR24 XT-HHT Pyrograf III; Applied Sciences Inc., USA) were suspended in pure acetone to produce coatings with $\mathrm{CNF} /(\mathrm{PVDF}$ +PMMA) wt. ratios of $0.068,0.138$, or 0.281 , and in 20/80 wt \% DMF/acetone mixtures for coatings with $\mathrm{CNF} /(\mathrm{PVDF}+\mathrm{PMMA})$ wt. ratios of $0.587,0.921,1.1 .^{2}$ Upon drying, the coatings display static water contact angles above $150^{\circ}$ (superhydrophobic) and droplet roll-off angles near or below $10^{\circ}$ indicating self-cleaning ability (i.e., water droplets roll off the inclined surface, thus removing impurities). The optimal wt. ratio of PTFE filler particles in the PVDF+PMMA polymer matrix for attaining super repellency (i.e., water contact angle $>150^{\circ}$ ) was found ${ }^{2}$ to be $\mathrm{PTFE} /(\mathrm{PVDF}+\mathrm{PMMA})=5.76$, and was kept fixed in the present study. The composite films displayed surface morphologies dominated by clusters of PTFE particles and CNFs in the polymer matrix [Fig. 1(a)]. This surface structure resulted in high liquid repellency and electrical conductivities spanning over six orders of magnitude for the following weight composition range: polymer matrix/PTFE/CNF $1 / 5.76 / 0.068-1.1 .^{2} \mathrm{I}-\mathrm{V}$ curves were generated to extract the electrical resistance of the coatings, which was then used to determine conductivity using the measured values of the

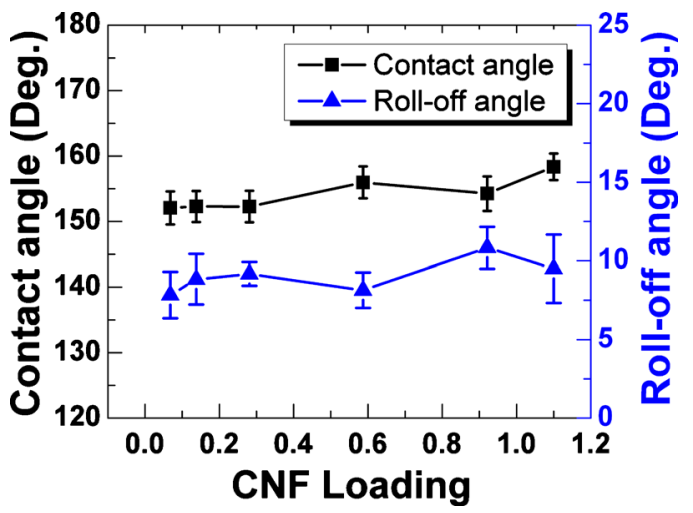

FIG. 2. (Color online) (a) Sessile water contact angle and roll-off angle for various (PVDF+PMMA)/PTFE/CNF composite coatings as a function of $\mathrm{CNF}$ loading expressed in terms of $\mathrm{CNF} /(\mathrm{PVDF}+\mathrm{PMMA})$ weight ratio. In all cases the PTFE/polymer weight ratio was 5.76.

FIG. 1. (Color online) (a) Scanning electron micrograph of a (PVDF+PMMA)/PTFE/CNF coating with high $\mathrm{CNF}$ loading (1.1) expressed as $\mathrm{CNF} /(\mathrm{PVDF}$ + PMMA) wt. ratio. (b) Electrical conductivity and average power transmission (EM frequencies in the range 570-630 GHz) for coatings with varying CNF loading. Power transmission in this frequency range decreases monotonically with CNF content. In all cases, the PTFE/polymer weight ratio was 5.76. The conductivity regimes suitable for different applications are separated by the horizontal line at $10 \mathrm{~S} / \mathrm{m}$. (c) A frequency domain $\mathrm{THz}$ spectroscopy system based on broadband quasioptical Schottky diode detectors. This system was used to quantify the power transmission curves shown in (b). Mirrors A and B collimate and then focus the beam through the sample. Mirrors C and D collimate the transmitted signal again and focus it onto the detector. The tested sample is mounted on a computercontrolled XY positioning stage, and placed at the focal point of the $\mathrm{THz}$ beam between mirrors $\mathrm{B}$ and $\mathrm{C}$. coating thickness, width and length. Electrical conductivity of the coatings rose with CNF loading [Fig. 1(b)].

The SE of such coatings in the $570-630 \mathrm{GHz}$ frequency range was measured by the frequency domain $\mathrm{THz}$ spectroscopy instrument ${ }^{11}$ shown in Fig. 1(c). The THz energy was coupled to a zero-bias Schottky diode broadband detector ${ }^{12}$ through four off-axis parabolic mirrors A-D [see Fig. 1(c)]. For two-dimensional (2D) mapping measurements, the source operated at one frequency, and the sample was scanned using a 2D positioning stage [Fig. 1(c)]. Averaged voltage response data was taken at each position and was then normalized to the detector response without sample. A 2D attenuation image was reconstructed electronically.

Water droplet contact and roll-off angle measurements were performed using a goniometer-type optical setup. ${ }^{13}$ The results are displayed in Fig. 2, where static water contact angles remain above $150^{\circ}$ for all CNF loadings. The corresponding contact angle for CNF-free coatings was $158^{\circ},^{2}$ indicating that super-repellency is not contingent upon the CNFs. Water droplet roll-off angles remained close or below $10^{\circ}$, confirming the self-cleaning nature of these coatings.

Figure 3 shows the $\mathrm{THz}$ power transmission for six samples with CNF content from 1 to 14 wt $\%$. The $\mathrm{THz}$

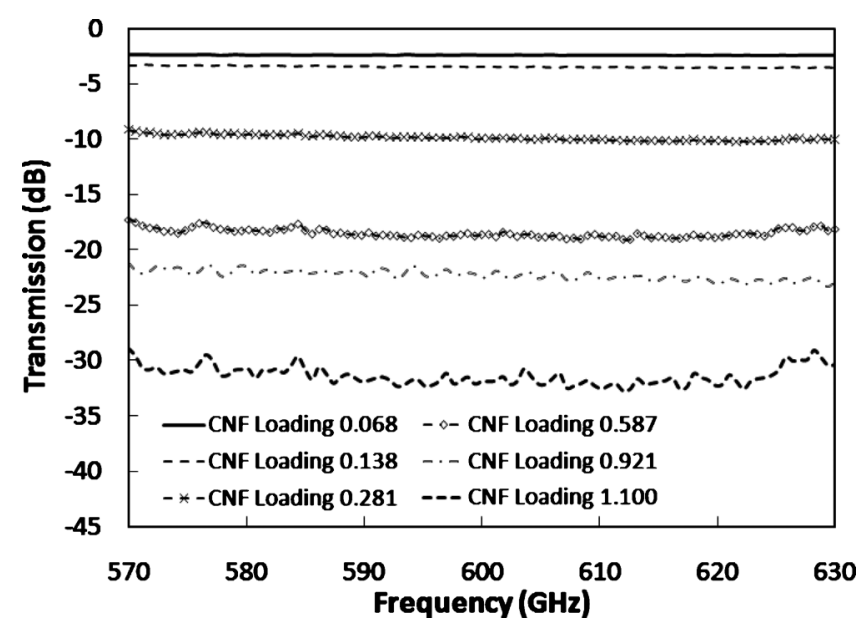

FIG. 3. Measured THz transmittance spectra for six coatings with varying CNF content (values in legend) in the frequency range $570-630 \mathrm{GHz}$. 


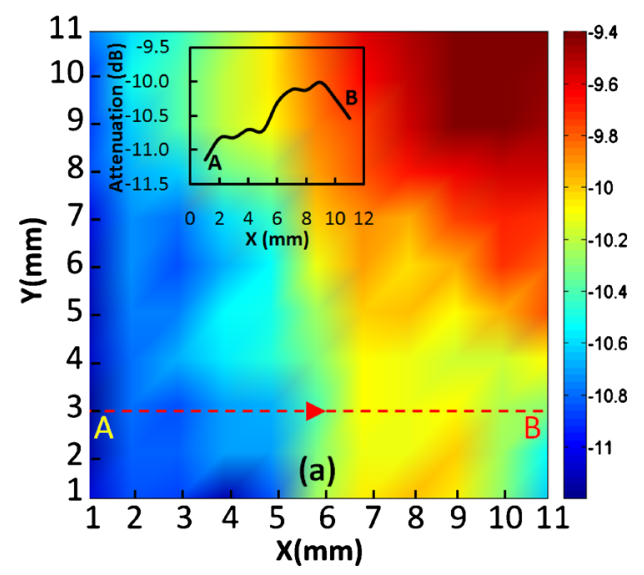

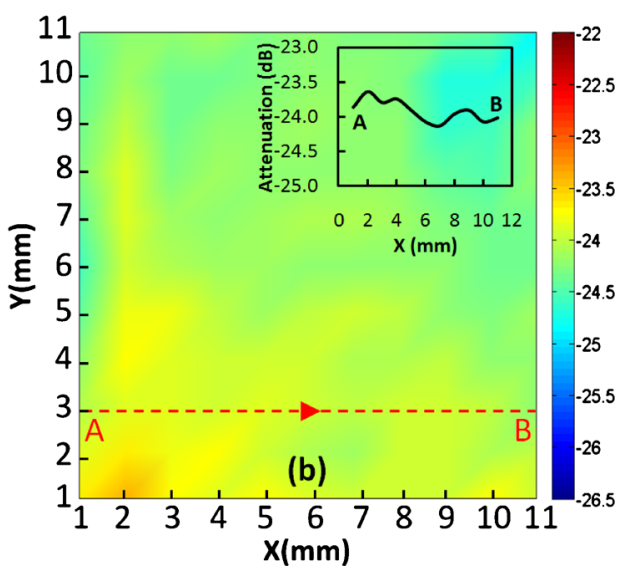

FIG. 4. (Color online) 2D distribution of $600 \mathrm{GHz}$ power attenuation (in decibel) for (a) coating with $\mathrm{CNF}$ loading 0.281 , and (b) coating with CNF loading 0.921, as measured using the frequency domain $\mathrm{THz}$ system of Fig. 1(c). The two plots show attenuation uniformity of $17.9 \%$ and $4.1 \%$, respectively, for each of the two samples. The insets show attenuation scans across $A B$ in each frame. transmittance (in decibels), or EMI SE is defined by SE $(d \mathrm{~B})=-10 \log _{10}\left(P_{\text {tran }} / P_{\text {inc }}\right)$, where $P_{\text {tran }}$ and $P_{\text {inc }}$ are transmitted and incident THz powers. The transmittance curves for the first five samples in Fig. 3 are quite uniform over the entire frequency range. The nonuniformities for the highest CNF loading are explained in Ref. 18. The spectra in Fig. 3 were averaged for each sample over the frequency range to produce the power transmission curve in Fig. 1(b). The gradually increasing average SE of 2.4 to $32.0 \mathrm{~dB}$ in Fig. 3 is consistent with the rising CNF content in these samples.

To study the coating uniformity and its effect on shielding property, we performed $2 \mathrm{D}$ scanning measurements for two samples (CNF loadings of $0.281,0.921$ ) at $600 \mathrm{GHz}$. The scanning area was $10 \times 10 \mathrm{~mm}^{2}$ and the scanning step size was $0.5 \mathrm{~mm}$. As shown in Fig. 4(a), the transmittance of the scanned region of the first sample (CNF loading 0.281) varied within $\sim 17.9 \%$. In comparison, the second sample (CNF loading 0.921) showed much better uniformity of $\sim 4.1 \%$ [Fig. 4(b)], which is satisfactory for practical applications. The uniformity of the remaining four samples was closer to the sample with CNF loading 0.921, indicating that spatial uniformity of decibel attenuation below $10 \%$ can be expected using the present method.

In principle, SE values higher than those in Fig. 3 could be attained with CNFs of higher conductivity. There exist different ways ${ }^{10}$ to improve the conductivity of vapor grown CNFs, such as acid treatment, carbonization, graphitization, open air etching, etc. Among these processes, graphitization is most effective. ${ }^{10}$ The CNFs employed in the present work have been treated at $3000{ }^{\circ} \mathrm{C}$, and were confirmed to have high electrical conductivity [Fig. 1(b)]. An additional factor contributing to high conductivity at low filler particle loadings is the high aspect ratio of the conductive filler, which lowers the percolation threshold. ${ }^{14}$ The CNFs used herein have very high aspect ratio $(>1300)$, which makes them a good choice as conductive fillers. Since most of the shielding is provided by the material up to a short depth from the surface (skin depth), fillers with high specific volume and surface area should display higher SE. ${ }^{10,15}$ The hollow cavity of the CNFs (see Ref. 18) not only increases their specific surface area and volume, but also enhances internal EM reflection, thus further contributing to their SE. ${ }^{16}$ Finally, the CNFs feature conductive elements spanning a wide range of length scales (see Ref. 18), which promotes broadband attenuation, ${ }^{17}$ as is evident from the fairly flat SE values (Fig. 3) in the range $570-630 \mathrm{GHz}$.

In conclusion, we studied the $\mathrm{THz}$ shielding and attenuation properties of polymer-based CNF-containing superhydrophobic large-area coatings, in the frequency range 570 $630 \mathrm{GHz}$. The THz SE of the CNF films rises with CNF wt. content, with a SE as high as $\sim 32 \mathrm{~dB}$ achieved at the highest possible CNF content (sprayability constraint). The spatial attenuation uniformity of these films at $600 \mathrm{GHz}$ showed good results for practical applications in future $\mathrm{THz}$ communication systems.

A.D. and L.L. contributed equally to this work. This work was partially supported by NSF Grant No. ECCS1002088.

${ }^{1}$ G. G. Tibbetts, M. L. Lake, K. L. Strong, and B. P. Rice, Compos. Sci. Technol. 67, 1709 (2007).

${ }^{2}$ A. Das, H. T. Hayvaci, M. K. Tiwari, I. S. Bayer, D. Erricolo, and C. M. Megaridis, J. Colloid Interface Sci. 353, 311 (2011).

${ }^{3}$ P. H. Siegel, IEEE Trans. Microwave Theory Tech. 50, 910 (2002).

${ }^{4}$ T. R. Globus, M. L. Norton, M. I. Lvovska, D. A. Gregg, T. B. Khromova, and B. L. Gelmont, IEEE Sens. J. 10, 410 (2010).

${ }^{5}$ E. R. Brown, E. A. Mendoza, D. Xia, and S. R. J. Brueck, IEEE Sens. J. 10, 755 (2010).

${ }^{6}$ T. G. Phillips and J. Keene, Proc. IEEE 80, 1662 (1992).

${ }^{7}$ L. Liu, H. Xu, A. W. Lichtenberger, and R. M. Weikle II, IEEE Trans. Microwave Theory Tech. 58, 1943 (2010).

${ }^{8}$ M. A. Seo, J. H. Yim, Y. H. Ahn, F. Rotermund, D. S. Kim, S. Lee, and H. Lim, Appl. Phys. Lett. 93, 231905 (2008).

${ }^{9}$ O. Shenderova, V. Grishko, G. Cunningham, S. Moseenkov, G. McGuire, and V. Kuznetsov, Diamond Relat. Mater. 17, 462 (2008).

${ }^{10}$ M. H. Al-Saleh and U. Sundararaj, Carbon 47, 2 (2009).

${ }^{11}$ L. Liu, J. L. Hesler, R. M. Weikle, T. Wang, P. Fay, and H. Xing, “A 570-630 GHz Frequenzy Domain Spectroscopy System Based on a Broadband Quasi-Optical Schottky Diode Detector," Int. J. High Speed Electron. Syst. (to be published).

${ }^{12}$ L. Liu, J. L. Hesler, H. Xu, A. W. Lichtenberger, and R. M. Weikle II, IEEE Microw. Wirel. Compon. Lett. 20, 504 (2010).

${ }^{13}$ M. K. Tiwari, I. S. Bayer, G. M. Jursich, T. M. Schutzius, and C. M. Megaridis, ACS Appl. Materials \& Interfaces 2, 1114 (2010).

${ }^{14}$ T. Prasse, J. Y. Cavaille, and W. Bauhofer, Compos. Sci. Technol. 63, 1835 (2003)

${ }^{15}$ R. M. Bagwell, J. M. McManaman, and R. C. Wetherhold, Compos. Sci. Technol. 66, 522 (2006).

${ }^{16}$ S. Y. Yang, K. Lozano, A. Lomeli, H. D. Foltz, and R. Jones, Composites, Part A 36, 691 (2005).

${ }^{17}$ V. L. Kuznetsov and Y. V. Butenko, in Synthesis, Properties and Applications of Ultrananocrystalline Diamond, NATO Science Series, edited by D. Gruen, O. Shenderova, and A. Vul (Springer, Amsterdam, 2005), p. 199.

${ }^{18}$ See supplementary material at http://dx.doi.org/10.1063/1.3583523 for HRTEM images of as-grown CNFs and their heat-treated derivatives. 